\title{
Barriers to use of simulation-based education
}

\section{[Les barrières à l'utilisation de la formation basée sur simulateur]}

Georges L. Savoldelli MD MEd, ${ }^{\dagger} \dagger$ Viren N. Naik MD MEd FRCPC, ${ }^{\dagger} \dagger$ Stanley J. Hamstra PhD, ${ }^{*} \ddagger$

Pamela J. Morgan MD CCFP FRCPC $\$$

Purpose: Barriers to simulation-based education in postgraduate and continuing education for anesthesiologists have not been well studied. We hypothesized that the level of training may influence attitudes towards simulation-based education and impact on the use of simulation. This study investigated this issue at the University of Toronto which possesses two sites equipped with high-fidelity patient simulators.

Methods: A 40-question survey of experiences, perceptions, motivations and perceived barriers to simulation-based education, was distributed to 154 anesthesiologists attending a departmental conference. Data were analyzed using descriptive statistics and associations between responses were assessed using either the Chi-Square statistic or a one-way analysis of variance.

Results: The rate of response was $58 \%$. Residents had experienced simulation-based education $(96 \%)$ more often than staff (58\%) and fellows (36\%), $(P<0.00$ I respectively). Residents had also attended more simulation sessions than staff and fellows (mean 2.8 vs 1.05 and 1.04, $P<0.00$ I respectively). Residents and fellows found simulation-based education more relevant for their training than staff ( $88 \%$ vs $65 \%, P<0.05$ ). Eighty-one percent of the respondents identified at least one significant barrier that prevents or limits them from attending simulator sessions. Staff anesthesiologists perceived multiple barriers and identified 'time' and 'financial issues' as significant barriers.

Conclusion: Anesthesiologists' level of training influences their attitudes towards and their perceptions of simulation-based education. This survey has identified perceived barriers that may limit a wider utilization of simulation. These results may be used to implement targeted actions such as course design, incentives, and information strategies, which could improve access and future use of simulation.
Objectif : Les barrières à la formation fondée sur la simulation en enseignement universitaire supérieur et en formation continue pour les anesthésiologistes ne sont pas bien connues. Nous avons émis l'hypothèse que le niveau de formation pouvait influencer l'attitude face à ce type de formation et avoir un effet sur l'utilisation de la simulation. Notre étude a été réalisée à l'université de Toronto qui possède deux sites équipés de simulateurs de haute fidélité.

Méthode : Un questionnaire de 40 questions sur les expériences, les perceptions, les motivations et les barrières perçues de la formation par simulation a été distribué à 154 anesthésiologistes en réunion départementale. Les données ont été analysées par des statistiques descriptives et les liens entre les réponses par le test chi-deux ou une analyse simple de la variance.

Résultats : Le taux de réponses a été de $58 \%$. Les résidents avaient plus d'expérience de la simulation (96\%) que les spécialistes (58\%) et les boursiers (36\%), ( $P<0,00$ I respectivement). Les résidents avaient aussi assisté à plus de sessions de simulation que les spécialistes et les boursiers (moyenne de 2,8 vs I,05 et I,04, $P<0,001$ respectivement). Les résidents et les boursiers ont trouvé la simulation plus pertinente que les anesthésiologistes (88\% vs $65 \%, P<0,05)$. Parmi les répondants, $81 \%$ ont reconnu au moins une barrière importante qui empêche ou limite la participation à des sessions de simulation. Les spécialistes ont perçu de multiples barrières significatives dont le «temps» et «les questions financières».

Conclusion : Le niveau de formation des anesthésiologistes influence l'attitude face à la simulation et leurs perceptions de la formation par simulation. Les limites à un plus grand usage de la simulation, reconnues dans l'enquête, pourraient servir à des interventions ciblées comme l'organisation d'un cours, des incitatifs et des stratégies d'informations pour améliorer l'accès à la simulation et son usage futur.

From the Wilson Center for Research in Education Faculty of Medicine; ${ }^{*}$ the Department of Anesthesia, $†$ St. Michael's Hospital; the Departments of Surgery, $\ddagger$ and Anesthesia, $\$$ Sunnybrook and Women's College Health Sciences Centre, University of Toronto, Toronto, Ontario, Canada.

Address correspondence to: Dr. Georges L Savoldelli, Department of Anesthesia, St. Michael's Hospital, 30 Bond Street, Toronto,

Ontario M5B 1W8, Canada. Phone: 416-864-5071; Fax: 416-864-6014; E-mail: georges.savoldelli@utoronto.ca

Reprints will not be available from the author.

Accepted for publication May 3, 2005.

Revision accepted June 3, 2005. 


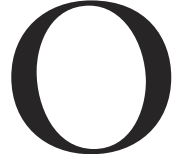

VER the last decade, there has been a tremendous growth in the use of highfidelity simulators worldwide. ${ }^{1}$ Many universities across North America and Europe are considering purchasing this technology or have recently acquired simulator facilities. Within the specialty of anesthesia, simulation has been used for various educational purposes, including anesthesia crisis resource management (ACRM), advanced life support algorithms, rare events, airway management, and technical skills to name a few. These education events exist for a wide range of levels of training and experience. ${ }^{1-6}$

Anesthesiologists find simulation learning experiences rewarding, valuable and likely to have positive influences on their clinical practice. ${ }^{7,8}$ At the same time, they also find this education stressful and intimidating. ${ }^{9}$ Interestingly, little is known about the motivations that attract them towards this learning modality or about the perceived barriers that may deter them from it. Studies on conventional continuing education have shown that factors such as age and position may influence anesthesiologists' attitudes and perceived barriers towards continuing education. ${ }^{10}$ Drawing from our personal experiences as educators involved in simulation, we hypothesized that these factors may similarly affect anesthesiologists' attitudes towards simulation-based education.

Considering the potential implications for the dissemination of this educational modality and the accessibility to simulation, we decided to test this hypothesis and conducted this survey at our university. Currently, the Department of Anesthesia at the University of Toronto benefits from two simulation centres fully equipped with high fidelity mannequins in a mock operating room. Since 1995, educational sessions have been offered to medical students, residents, academic anesthesiologists, and communitybased anesthesiologists. The purpose of this study was to investigate anesthesiologists' previous experiences, perceptions, motivations and perceived barriers related to simulation as an educational modality, and to determine if the level of training of the responders influences their opinions.

\section{Methods}

Following Institutional Research Ethics Board approval, a 40 -item questionnaire was distributed to 154 anesthesiologists attending a departmental conference in 2004. The participants were staff, fellows and residents currently working in all hospitals affiliated with the University of Toronto. This method of sampling was chosen because it was felt that it could guarantee

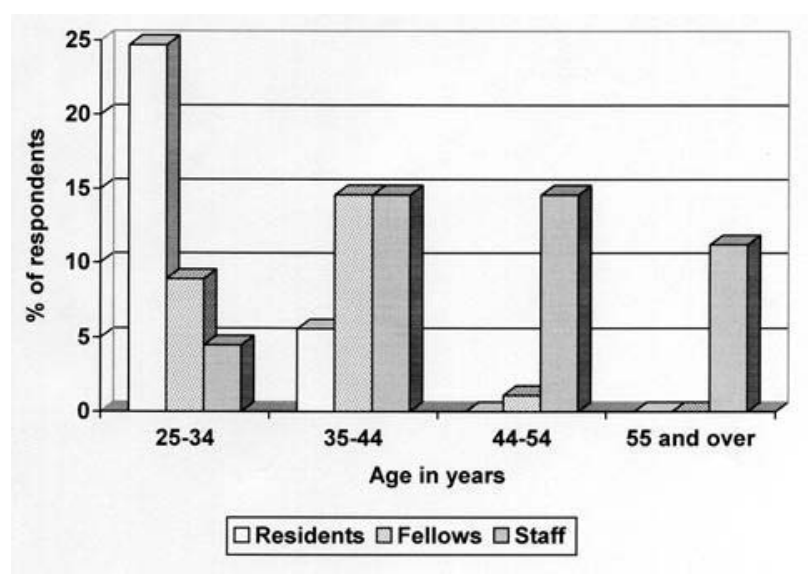

FIGURE Age distribution according to the level of training.

a good representation of every level of training in a convenient way.

The survey was developed by the authors and piloted on ten different occasions to guarantee clarity and absence of ambiguity of the questions. Answers to open-ended questions in the pilot surveys were also used to generate additional closed questions for the final survey. The questionnaire was self-administered and anonymous and was designed to gather information regarding demographics, previous experiences, perceptions, motivation and perceived barriers related to simulation as an educational modality (Appendix, available as Additional Material at cja-jca.org).

Data were entered into a spreadsheet and analyzed using SSPS 10.0 (Chicago, IL, USA). Traditional descriptive statistics were used to examine the data and the responses to various questions were cross-tabulated. Responses derived from five point Likert scales ( 1 = strongly disagree; 2 = disagree; $3=$ undecided; 4 $=$ agree; $5=$ strongly agree) were classified into three categories: either disagree ( 1 or 2 ), undecided (3), or agree (4 or 5 ). Associations between responses to different questions were assessed using either the Chi-square statistic or a one-way analysis of variance, depending on the nature of scale. A $P<0.05$ was considered significant.

\section{Results}

Responses and demographics

Forty staff anesthesiologists, 22 fellows, and 27 residents returned the questionnaire for a response rate of $58 \%$. The Figure summarizes the age distribution of the 
TABLE I Experience and participation in simulation education according to the level of training

\begin{tabular}{lllll}
\hline & Residents & Fellows & Staff & Significance \\
\hline Respondents who had participated in simulation training (\%) & 98 & 36 & 58 & $P<0.001\left(\chi^{2}\right)$ \\
Respondents who had participated in simulation within the last 2 yr (\%) & 93 & 27 & 15 & $P<0.001\left(\chi^{2}\right)$ \\
Number of simulation sessions attended (mean \pm SD) & $2.8 \pm 1.4$ & $1.0 \pm 1.9$ & $1.1 \pm 1.3$ & $P<0.001$ \\
& & & & (ANOVA) \\
\hline
\end{tabular}

TABLE II Proportion of responders who classify a given course content as a high priority

$\begin{array}{ll}\text { Opportunities to manage rare events (e.g., malignant hyperthermia, anaphylaxis) } & 81 \% \\ \text { Teaching non-technical skills (ACRM principles, decision making) } & 62 \% \\ \text { Practicing and learning guidelines and algorithms (airways, ACLS, ATLS) } & 61 \% \\ \text { Fostering teamwork and involving other professionals (surgeons, nurses, etc.) } & 48 \% \\ \text { Teaching technical skills (e.g., airways, chest tubes, etc.) } & 25 \%\end{array}$

Proportions show overall responses as there was no difference between faculty, residents or fellows. ACRM $=$ anesthesia crisis resource management; ACLS = advanced cardiac life support; ATLS = advanced trauma life support.

respondents according to their level of training. The proportion of female responders among residents, fellows and staff was 30, 27 and 33\% respectively. In our department, the average age of residents, fellows, and staff is 31,35 , and 46 respectively, and the proportion of women is 29,27 , and $30 \%$ respectively. Therefore, in terms of age and gender, the sample was found to be representative of the study population. With one exception, all responders were based predominantly in a university-based practice within the last year. Foci of anesthesia practice consisted of all types of surgical intervention $(74 \%)$, specialized anesthesia $(24 \%)$, intensive care $(6 \%)$, and pain management $(9 \%)$. In addition to their clinical activity, $78 \%$ of the staff, $27 \%$ of the fellows, and $11 \%$ of the residents reported at least one other academic responsibility (i.e., involvement in administration, research, or education).

\section{Experience and participation in simulation education}

Table I summarizes responders' experiences and participation in simulation-based education stratified by their level of training. Overall, residents were more likely to have participated in simulation-based training and experienced simulation more often and recently than staff and fellows. Amongst the responders who had participated in simulation-based education, the vast majority (92\%) agreed that its educational value was good and that it was an enjoyable experience $(86 \%)$. However, only one third thought that simulation training had influenced their clinical practice, with the majority being undecided.
What type of simulation-based education is relevant to anesthesiologists?

Five possible "course content" items were suggested. Responders were asked to prioritize these suggestions according to their relevance for their own education. A four-point rating scale $(1=$ not a priority, $2=$ low priority, $3=$ medium priority, $4=$ high priority) was used. The proportion of responders who classified a given course content as a high priority is displayed in Table II. There was no difference in course content prioritization between faculty, residents or fellows.

\section{Perceptions of simulation-based training and willing-} ness to go or return to the simulation centre

Overall the majority of the responders agreed that simulation may contribute to patient safety $(85 \%)$, is a useful educational technology (85\%), and offers advantages over more traditional training (79\%). There was a significant association between holding a trainee position (resident or fellow) and 1) willingness to go or return to the simulation centre (trainees $90 \%$ vs staff $68 \%, P<0.05), 2$ ) finding simulation-based education relevant for one's own training (trainees $88 \%$ vs staff $65 \%, P<0.05$ ), and 3 ) supporting the mandatory use of simulation during residency (trainees $84 \%$ vs staff $58 \%, P<0.05$ ).

Simulation and continuing medical education (CME) Two-thirds of the responders (including $58 \%$ of the staff) agreed that simulation should be recommended for CME, while 26\% were undecided. However, less than half of the staff $(45 \%)$ were aware that Maintenance of Certification credits (MainCert credits) are currently granted when attending simulation- 
TABLE III Proportion of responders who selected the suggested reasons to the question: "What would increase your likelihood of attending a simulation-based course?"

\begin{tabular}{lc}
\hline The provision of an individual evaluation profile (strengths and weaknesses) for my personal use & $64 \%$ \\
If the course were given in my own hospital (e.g., mobile simulation centre in a trailer) & $48 \%$ \\
The provision of a certificate that would reduce malpractice insurance premium & $43 \%$ \\
If more MainCert credits were given per hour of simulation time & $36 \% *$ \\
The provision of a ranking that compares my performances to others (for my personal use) & $34 \%$ \\
Others (open ended comment) & $10 \%$ \\
\hline
\end{tabular}

${ }^{*}$ Associated with holding a staff position $(P<0.05(\chi 2)$. MainCert $=$ Maintenance of Certification credits.

TABLE IV Proportion of staff and trainees (residents and fellows combined) who perceived barriers to simulation-based training and association with the level of training

\begin{tabular}{llll}
\hline Perceived barrier & Staff $(n=40)$ & Trainees $(n=49)$ & Significance $(\chi 2)$ \\
\hline Perceive at least one barrier (\%) & 90 & 73 & $P<0.05$ \\
Number of perceived barriers (mean \pm SD) & $1.8 \pm 1.1$ & $1.3 \pm 1.2$ & NS* \\
"Lack of free time" (\%) & 55 & 33 & $P<0.05$ \\
"Financial consequences of missing work" (\%) & 18 & 0 & $P<0.01$ \\
"Lack of training opportunities" (\%) & 23 & 39 & $\mathrm{NS}$ \\
"Stressful/intimidating environment" (\%) & 25 & 22 & $\mathrm{NS}$ \\
"Fear of educator's/peer's judgments" (\%) & 25 & 18 & $\mathrm{NS}$ \\
"Fear of inaccurate reflection of clinical ability" (\%) & 25 & $\mathrm{NS}$ & $\mathrm{NS}$ \\
"Distance to simulation centre" (\%) & 8 & 8 & \\
\hline
\end{tabular}

NS = not significant. *Comparison performed by ANOVA.

based education.

\section{Potential incentives and motivations}

Five possible reasons were suggested as potential motivations or incentives to increase the likelihood of attending a simulator-based course. Table III shows the proportion of responders who selected the various responses. Except for the provision of more MainCert credits, which was associated with holding a staff position $(P<0.05)$, no other significant association with the level of training was present. In the open-ended comments, $10 \%$ of the responders mentioned other potential incentives which varied from "having time booked for it", "receiving financial compensation", to "increased accessibility and course offering".

\section{Perceived barriers}

Respondents were asked if they perceived significant barriers that prevent or limit them from pursuing simulator-based education. A list of nine potential barriers, including a "none" and an "other/open ended" category was suggested. Eighty-one percent of the respondents identified at least one significant barrier. Compared with trainees, staff anesthesiologists were more likely to perceive at least one barrier $(P<0.05)$ and although not significant, on average they perceived more barriers. Holding a staff position was also significantly associated with considering "lack of free time" and "financial consequences of missing work" as a barrier. The lack of "publicized courses" and "policy promoting this type of education" were mentioned in the open-ended comments section. Table IV summarizes the type of barriers and their association with the level of training.

\section{Discussion}

Our results indicate that anesthesiologists value simulation-based education. However, they perceive barriers to this type of training. A significant proportion of the responders feared educators' or peers' judgments and were concerned by the stressful and intimidating environment created in the simulator setting. Compared with trainees, staff anesthesiologists had less experience with simulators, found it less relevant for their current training, and perceived more barriers.

Simulation, using high-fidelity mannequins in a realistic environment, is being increasingly used in undergraduate, postgraduate and CME. ${ }^{3,6,11-15}$ Anesthesia has been and remains among the leading specialties in the field, and the number of anesthesia simulation centres worldwide is increasing exponentially. ${ }^{1}$ The University of Toronto has over a decade of experience with simulation based education. Ten years ago, when simulation was emerging in North America, Kurrek et al. surveyed anesthesiologists' opinions. ${ }^{9}$ They showed that despite significant antic- 
ipated anxiety, anesthesiologists were enthusiastic about the use of simulation. However, a large majority of the responders had not been exposed to the simulation environment. Interestingly, staff anesthesiologists that had taken the ACRM course at the time recommended that it should be taken on average every 18 months. Ten years later, this survey suggests that simulation has largely failed to establish itself as a regular educational activity at the CME level. Our results show that although there is general agreement that simulation should be part of CME, simulation is not a regular CME activity among staff anesthesiologists. Only $58 \%$ of the staff had ever participated in simulation-based training, and only $15 \%$ participated within the last two years. Conversely, simulation appears very well entrenched during residency training. One explanation for the difference in participation is that simulation was not found to be as relevant for staff as it is for trainees ( $65 \%$ and $88 \%$ respectively, $P<0.05$ ). However, both faculty and trainees identified similar course content as relevant to their education.

The reasons to participate in educational activities differ between staff and trainees. For faculty, the relevance for clinical practice and the need to keep up to date with new technology are important motivations at the CME level. ${ }^{10,11,16}$ Simulation has the potential to meet these needs. As educators, our role is to provide course content and learning objectives that are relevant and adapted to the level of training of the learners. Simulation is such a versatile educational tool, that the way we use it is even more important than the tool itself. In ACRM type courses for example, the emphasis is put on non-technical skills that have been identified as major determinants of successful anesthesia crisis management. ${ }^{17}$ These skills encompass cognitive skills and interpersonal skills that are not necessarily acquired and practiced through routine clinical experience. Ideally, they should be specifically taught and regularly practiced by anesthesiologists independent of their level of training. ${ }^{5}$ Simulated scenarios followed by reflection, feedback, and the opportunity to practice again, offer the ideal environment to teach and refine those skills. Equally relevant for the fully trained anesthesiologist is the fact that the simulation room can be used as a risk free environment for interactive, hands-on training involving the use of new medical equipment, devices, or drugs. Therefore, there is no reason why simulation should be less relevant for the continuing education of more experienced anesthesiologists.

Another important difference between a trainee and a staff anesthesiologist, in terms of education, is that while the former follows a predefined cur- riculum, the latter must plan his/her own continuing education according to individual needs. Simulationbased education is mandatory at our university during undergraduate clerkship rotation and during residency, but it is completely optional at the CME level. Our findings that more residents have participated in simulation are therefore not surprising. At the CME level, the learner has a great deal of autonomy and choice. In this context, age and familiarity can influence the type of CME activity and it has been suggested that perceived barriers may impact continuing training. ${ }^{10,16}$ In our survey, staff were not only older but they also perceived more barriers than trainees. It is possible that these barriers, combined with the absence of mandatory participation at the CME level, have contributed to the relative lack of success of simulation for CME.

This study is the first to specifically explore the perceived barriers to simulation among anesthesiologists. The most common barrier for staff was the "lack of free time". The most common barrier for trainees was the lack of training opportunity, even though they had participated more often in simulation-based education than staff. Compared with trainees, staff clearly perceived more barriers, and not surprisingly identified "lack of free time" and "financial issues" as significant barriers.

Other perceived barriers included the stressful and intimidating environment, the fear of educator's or peer's judgment, and the fear of an inaccurate reflection of one's own clinical ability. It is recognized that a simulated scenario may be stressful, may trigger strong emotions, and may increase the number of errors committed by the participant. The issues of having one's performance analyzed and reflected upon from a critical perspective can be very daunting. Nonetheless, this ability highlights the power and the strength of high-fidelity simulation. Simulation sessions provide the venue to commit errors and to reflect on them. ${ }^{15}$ Since stress and emotions are generated, the overall learning experience is potentially more intense and more effective. Recognizing those assets is relatively easy, but implementing them successfully and getting them accepted are more difficult. This issue is at the heart of a much broader problem within the health care system. In fact, it illustrates how difficult it is to move away from an inherited culture of silence and blame when an error is made to a new "culture of safety", where error disclosure and error analysis are central tenets. ${ }^{15,18}$ It has been argued that simulation-based education has the potential to promote this new culture. ${ }^{7,15,18}$ Unfortunately, our results suggest that the existing culture threatens the 
acceptance of simulation-based education. The implementation of simulation-based education should ideally be part of a system and organizational approach to quality improvement and patient safety, where both approaches reinforce and nurture the other.

This type of cultural change is a slow process, but the type of education one receives can influence it favourably. It is therefore possible that as residents and younger trainees experience high-fidelity simulation, they may be more amenable to continuing education when they become staff. They may also encourage others to participate if their experiences in simulation are positive.

Current access to simulation could also be improved through incentives. In Boston, anesthesiologists are now eligible for malpractice premium reductions if they have participated in a faculty ACRM course. ${ }^{3}$ This type of incentive may be less attractive in countries such as Canada, where insurance premiums are lower. Nevertheless, $43 \%$ of the responders would increase their participation if it were offered.

Surprisingly, the most compelling potential incentive was the provision of an individual evaluation profile (strengths and weaknesses) for personal use. It is somehow paradoxical that participants fear educators' judgment and making errors, while at the same time many acknowledge that a formative evaluation with the provision of an evaluation profile would be useful. A current trend in simulation that could partially address this concern is to move away from an individual performing during a scenario, and focus more on team training. In this context, team performance is examined rather than the critical analysis of one individual; thus, the debriefing may be less intimidating. However, it is unknown whether team debriefing is as effective as individual feedback, and would still be considered a valuable incentive.

Our study has several limitations. It was conducted at one university where the exposure to simulation is probably greater than at other centres across Canada. It included only academic anesthesiologists, fellows, and residents. Hence, the generalizability of the results to other universities, or to communitybased practitioners is unknown. Although our rate of response was good for a "single shot" survey, our sample size did not allow us for example to analyze the responses according to the age of the responders. Finally, we did not combine our quantitative approach with a qualitative survey that could have provided a richer perspective on the perceived barriers.

Overall, our results have provided a better understanding of anesthesiologists' perceptions, motivations, and perceived barriers towards simulation-based education in a university setting. These results may help in developing targeted actions such as improved course design, incentives, and marketing strategies, which could improve access and future utilization of simulation. Better access to simulator-based education may translate into enhanced performances in the clinical setting, and potentially improve patient safety.

\section{References}

1 Morgan PJ, Cleave-Hogg D. A worldwide survey of the use of simulation in anesthesia. Can J Anesth 2002; 49: 659-62.

2 Gaba DM. Improving anesthesiologists' performance by simulating reality. Anesthesiology 1992; 76: 491-4.

3 Blum RH, Raemer DB, Carroll JS, Sunder N, Feinstein $D M$, Cooper JB. Crisis resource management training for an anaesthesia faculty: a new approach to continuing education. Med Educ 2004; 38: 45-55.

4 Glavin R, Maran N. An introduction to simulation in anaesthesia. In: Greaves JD, Dodds C, Kumar CM, Mets B (Eds). Clinical Teaching: A Guide to Teaching Practical Anaesthesia. Lisse, The Netherlands: Swets \& Zeitlinger; 2003: 197-205.

5 Howard SK, Gaba DM, Fish KJ, Yang G, Sarnquist FH. Anesthesia crisis resource management training: teaching anesthesiologists to handle critical incidents. Aviat Space Environ Med 1992; 63: 763-70.

6 Byrick R, Cleave-Hogg D, McKnight D. A crisis management program for residents in anesthesia. Acad Med 1998; 73: 592.

7 Garden A, Robinson B, Weller J, Wilson L, Crone D. Education to address medical error-a role for high fidelity patient simulation. N Z Med J 2002; 115 : 133-4.

8 Weller J, Wilson L, Robinson B. Survey of change in practice following simulation-based training in crisis management. Anaesthesia 2003; 58: 471-9.

9 Kurrek MM, Fish KJ. Anaesthesia crisis resource management training: an intimidating concept, a rewarding experience. Can J Anaesth 1996; 43: 430-4.

10 Chambers WA, Ferguson K, Prescott GJ. Continuing medical education by anaesthetists in Scotland: activities, motivation and barriers. Anaesthesia 2000; 55: 1192-7.

11 Tetzlaff JE, Schoenwald P, Jackman D, Smith J. Continuing medical education and the anesthesiologist. J Clin Anesth 1999; 11: 164-72.

12 Morgan PJ, Cleave-Hogg D, DeSousa S, Tarshis J. Identification of gaps in the achievement of undergraduate anesthesia educational objectives using high-fidelity patient simulation. Anesth Analg 2003; 97: 1690-4.

13 Issenberg SB, Pringle S, Harden RM, Khogah S, Gordon $M S$. Adoption and integration of simulation-based 
learning technologies into the curriculum of a UK Undergraduate Education Programme. Med Educ 2003; 37(Suppl 1): 42-9.

14 Wong $A K$. Full scale computer simulators in anesthesia training and evaluation. Can J Anesth 2004; 51: 45564.

15 Ziv A, Wolpe PR, Small SD, Glick S. Simulation-based medical education: an ethical imperative. Acad Med 2003; 78: 783-8.

16 Weller J, Harrison M. Continuing education and New Zealand anaesthetists: an analysis of current practice and future needs. Anaesth Intensive Care 2004; 32: 59-65.

17 Fletcher GC, McGeorge P, Flin RH, Glavin RJ, Maran NJ. The role of non-technical skills in anaesthesia: a review of current literature. Br J Anaesth 2002; 88: 418-29.

18 Kohn LT, Corrigan JM, Donaldson MS; Committee on Quality of Health Care in America. To Err Is Human: Building a Safer Health System. Institute of Medicine. Washington, DC: National Academy Press; 1999. 